\title{
Usefulness of cardiac resynchronisation therapy devices and implantable cardioverter defibrillators in the treatment of heart failure due to severe systolic dysfunction: systematic review of clinical trials and network meta-analysis
}

\author{
M A García García, ${ }_{1}^{1}$ M A Rosero Arenas, ${ }^{2}$ R Ruiz Granell, ${ }^{3}$ F J Chorro Gascó, ${ }^{3,4}$ \\ A Martínez Cornejo ${ }^{1}$
}

- Additional material is available. To view please visit the journal online (http://dx. doi.org/10.1136/heartasia2015-010634).

${ }^{1}$ ICU Sagunto Hospital (Valencia), Sagunto, Valencia, Spain

${ }^{2} \mathrm{C}$. Salud Cheste (Valencia), Cheste, Valencia, Spain

${ }^{3}$ Cardiology Service Valencia University Hospital

${ }^{4}$ Faculty of Medicine, Department of Medicine, Valencia University. INCLIVA (Research Foundation of the Clinical Hospital of Valencia)

\section{Correspondence to}

M Á García García, Intensive Care Unit, Sagunto Hospital, Avda Dr Peset Aleixandre $n^{\circ}$ 81 puerta 21, Sagunto, Valencia 46009, Spain; mangelesymangel@hotmail. com

Received 27 April 2015 Revised 24 November 2015 Accepted 15 December 2015

\section{ABSTRACT}

Aim To assess the effectiveness of cardiac resynchronisation therapy (CRT), implantable cardioverter defibrillator (ICD) therapy, and the combination of these devices $(C R T+I C D)$ in adult patients with left ventricular dysfunction and symptomatic heart failure.

Methods A comprehensive systematic review of randomised clinical trials was conducted. Several electronic databases (PubMed, Embase, Ovid, Cochrane, ClinicalTrials.gov) were reviewed. The mortality rates between treatments were compared. A network was established comparing the various options, and direct, indirect and mixed comparisons were made using multivariate meta-regression. The degree of clinical and statistical homogeneity was assessed.

Results 43 trials involving 13017 patients were reviewed. Resynchronisation therapy, defibrillators, and combined devices (CRT+ICD) are clearly beneficial compared to optimal medical treatment, showing clear benefit in all of these cases. In a theoretical order of efficiency, the first option is combined therapy (CRT $+(C D)$, the second is CRT, and the third is defibrillator implantation (ICD). Given the observational nature of these comparisons, and the importance of the overlapping $\mathrm{Cls}$, we cannot state that the combined option (CRT+ICD) offers superior survival benefit compared to the other two options.

Conclusions The combined option of CRT+ICD seems to be better than the option of CRT alone, although no clear improvement in survival was found for the combined option. It would be advisable to perform a direct comparative study of these two options.

\section{INTRODUCTION}

Heart failure (HF) is a chronic clinical syndrome, caused by functional disorders of the heart, especially of the left ventricle (LV), resulting in dyspnoea on exertion and even at rest. It is one of the cardiovascular diseases responsible for increasing morbidity and mortality in developed countries, resulting in a significant economic impact. With an estimated survival rate of $50 \%$ at 5 years, HF is the first cause of hospital admissions among the elderly. All of this occurs despite the fact that in recent years many pharmacological treatments have been developed that have greatly improved the prognosis for patients with HF. ${ }^{1}$
Cardiac resynchronisation therapy (CRT) is a therapeutic option in patients with moderate to severe HF. It is able to correct ventricular electromechanical asynchrony, acting on the atrioventricular, ventricular, intraventricular, and intramural delay. ${ }^{2}$ It also produces reverse remodelling of the $\mathrm{LV}$, thus increasing its ejection fraction (LVEF) and decreasing the severity of associated mitral regurgitation. All the above translates into better tolerance to effort, an improvement in functional class, a reduction in the number of hospital admissions, an improvement in the quality of life, and also a reduction in mortality.

The spectrum of patients who benefit from this treatment is outlined in the recommendations given by scientific societies. The most common indication is for outpatients with a dilated cardiomyopathy with reduced LVEF, a QRS width $\geq 120$ ms (especially with a complete left bundle branch block), sinus rhythm, and New York Heart Association (NYHA) functional class III-IV, which continues to be symptomatic despite optimal medical and pharmacological treatment (OMT) (indication I, level of evidence A). ${ }^{3}$

Sudden cardiac death (SCD) occurs in approximately $2 \%$ of adults. It is believed that $>80 \%$ of these episodes are due to ventricular tachyarrhythmia. ${ }^{4}$ Patients with HF have a risk of SCD five times greater than the general population ${ }^{5}$; several published series ${ }^{5} 6$ show that $30-50 \%$ of cardiac deaths in patients with HF may be attributed to SCD. Clinical guidelines describe the efficiency of implantable cardioverter defibrillators (ICD) in the improvement of mortality by enabling the early interruption of these arrhythmias, both in patients who have suffered SCD who have been resuscitated (secondary prevention) and in those with a high risk of suffering from the condition (primary prevention).

Therefore, there is a group of patients with moderate-severe HF, with a depression of LVEF, and with no history of previous severe ventricular arrhythmia/SCD, who may benefit from these devices (CRT and ICD), either alone or in combination. The decision to utilise either of these technologies must be made based on efficacy assessment using rigorous tests. Previous meta-analyses have evaluated the efficacy/safety of a treatment in relation to a single comparator. As there is no 
information from clinical trials with direct comparisons of several treatment options, network meta-analyses are being used with increasing frequency which enable estimates to be made of the relative effect of various treatments via indirect comparisons. One advantage of these techniques is that when direct evidence is limited or insufficient, answers to clinically important questions can be given with all these tests evaluated together. Therefore they are an extension of classic meta-analyses, in which the assessment of direct comparison studies is carried out in addition to a complete evaluation of the information available for direct and indirect comparisons between treatments, thus increasing the statistical power of the estimates generated. ${ }^{78}$

To conduct indirect analysis we must verify several conditions. Transitivity, more than similarity of all the features in the studies, assumes that these studies are comparable because they do not differ as far as distribution of effect-modifying factors between two direct comparisons. This assumption that transitivity may break down treatments has been applied to different therapeutic indications. ${ }^{8} 9$ Consistency is another important aspect; it is the level of agreement in a closed cycle or loop between the estimates of results obtained in direct and indirect comparisons. There must be no differences in combined studies in the distribution of effect-modifying factors for outcomes to be consistent. It can be evaluated statistically.

The scant or non-existent studies evaluating some branches of treatment (CRT compared to ICD, or CRT + ICD compared to CRT), already mentioned in other similar studies, ${ }^{9}$ compels us to evaluate indirect and combined comparisons of treatments within a network meta-analyses. Our study attempts to assess the comparative efficacy of these treatments, both with each other and compared to OMT, and may serve as a guide for doctors in their day-to-day decision making concerning which type of device is suitable for the treatment of a specific patient.

\section{METHODS}

Our objective was to assess the efficacy of treatment with CRT and/or ICD in reducing patient mortality with symptomatic HF. A broad search in several databases was carried out, in order to find clinical trials in which these two devices were compared, singly or combined, either with one other or with OMT.

A comprehensive systematic review was conducted according to the recommendations concerning design quality of the PRISMA (Preferred Reporting Items for Systematic Reviews and Meta-Analyses) statement, ${ }^{10}$ and also following the recommendations of the Guidelines for Drawing Up and Critical Evaluation of a network meta-analysis proposed by Catalá-López et al. ${ }^{8}$

\section{Selection criteria}

Randomised clinical trials with the following elements were sought: population (patients with symptomatic $\mathrm{HF}$ and depressed LVEF, usually with wide QRS and/or echocardiographic asynchrony), and intervention (CRT, ICD or CRT + ICD devices, or OMT). There were several inclusion/exclusion criteria: restrict the search to humans; refer predominantly to adults (ie, exclude from this study congenital cardiomyopathies with instrumental management in early life); and have no limits on language and date of publication.

We rejected studies on secondary prevention of SCD with ICD therapy. Our interest was in patients at risk of suffering arrhythmia; therefore, the indication of these devices is primary prevention.

Several electronic databases were consulted: PubMed, with a broad strategy and search syntax: (('Electric Stimulation
Therapy'[Majr] OR 'Pacemaker, Artificial'[Majr] OR 'Defibrillators, Implantable'[Majr]) AND ('Heart Failure/ therapy'[Majr] OR 'Ventricular Dysfunction/therapy[Majr])) AND (Clinical Trial[ptyp] OR Meta-Analysis[ptyp] OR Randomised Controlled Trial[ptyp] OR Review[ptyp]), complemented with the 'broad' methodological filter option 'Clinical Queries'; Embase; Ovid; ClinicalTrials.gov; Cochrane Library, with DARE database (systematic reviews) AND CENTRAL database (clinical trials); Web of Knowledge; Trip Database; and two recent and powerful metabrowsers: EBSCOhost and Elsevier's SciVerse Hub. All this information was supplemented with bibliographic references in several published systematic reviews and meta-analyses, and experts in this field (RRG, FJCG) were consulted to search for unpublished studies. We searched for the results of completed works referred to in ClinicalTrials.gov.

All these databases were reviewed for the last time in October 2012. Constant review of the topic, and the receipt of email alerts from PubMed, enabled us to receive some additional studies published in 2013, which it was decided should be included in the study.

\section{Data abstraction and outcomes}

Basic information (population, intervention, and outcome) and methodological quality (risk of bias) were obtained from the finally accepted studies in a peer reviewed process (conducted by MAGG and MARA); in areas where there was no coincidence, there was discussion until agreement was reached.

Efficacy of ICD is measured only with mortality. The studies with other devices recorded other details (hospital readmission, quality of life, etc). Consequently, in this joint therapy assessment study (CRT and/or ICD) the measured outcome was mortality. This dichotomous variable was evaluated with an odds ratio (OR). Since the heterogeneity between studies was probable, the random effects model was assumed, and calculations were made using the DerSimonian and Laird model. Calculations were made using the Cochrane Collaboration RevMan 5.2 programme, the calculators made in Excel by Dr Joaquín Primo (available at the website http://www.redcaspe.org) and with STATA V.12.0.

We included data from crossover studies. These studies are usually shorter, and also report mortality of their patients. We can have efficacy data after the first period of active treatment, or at the end of the second period. As recommended by Cochrane Collaboration, we obtained a global estimation of the effect according to the results provided by the authors, ie, data at the end of the first period of treatment in some studies and data at the end of the second period in others.

\section{Methodological assessment}

The quality of these studies was assessed based on five items included in the bias assessment tool of the RevMan programme: generation of randomisation sequence, concealment of randomisation sequence (both define the selection bias), patient and doctor blinding (design bias), assessment of outcomes blinding (detection bias), and incomplete follow-up (loss bias). Each area was defined as low, high or unclear bias risk, and it was numerically qualified as $+1,-1$ or 0 ; the sum of these five numbers can provide a numerical estimate of the quality of the study. Studies with a score $\geq 3$ were deemed to be of good quality. This quality assessment is similar to the quantification of items on the classic Jadad scale, ${ }^{11}$ and it includes blinding in the assessment of the events. 


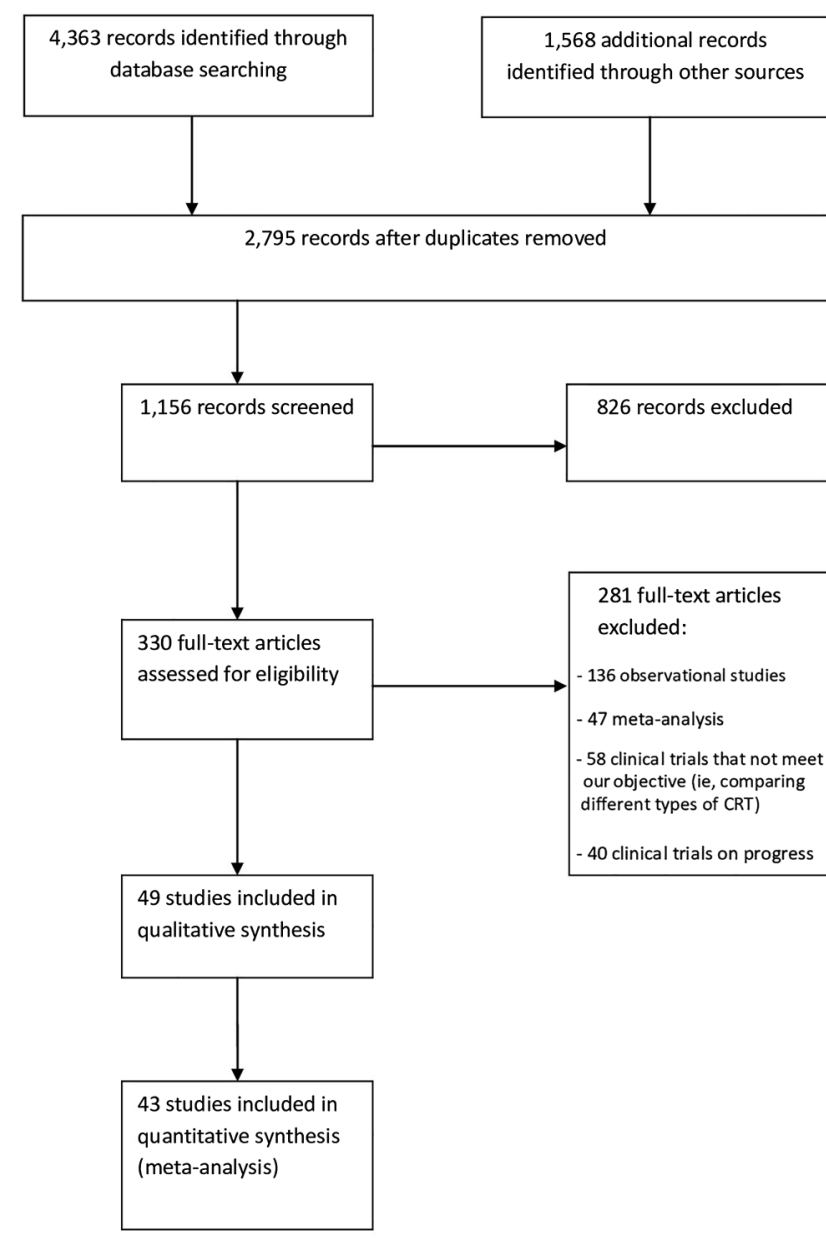

Figure 1 Flow chart of studies evaluated at different stages of the work. CRT, cardiac resynchronisation therapy.

\section{Statistical analysis}

Publication bias was assessed graphically, using a funnel plot (the studies are represented as points whose coordinates are the accuracy of the estimate and the effect estimate), and numerically, using the Begg and Egger methods; the number of unpublished clinical trials was also estimated (Gleser-Olkin and Rosenthal methods).

We assessed the degree of statistical and clinical heterogeneity of the meta-analysis. The statistical heterogeneity was globally measured with the Cochran's Q and $\mathrm{I}^{2}$ tests, and with graphic representations because of the lack of statistical power of both tests; L'Abbé method represents the rate of events of the treatment group compared to the rate of the control group, with the area of the estimated effect directly proportional to its total sample size. We also performed a subgroup analysis evaluating clinically important variables (previous vs not previous pacemaker, crossover vs non-crossover design, and other). Some degree of heterogeneity can be seen in many forest plots if the result of a study is different from the rest. We carried out an analysis of sensibility, making a new assessment of the effect excluding the different study, and seeing if there was a significant difference in the result.

Efficacy was assessed (measured as a reduction in mortality) in the studies which compared treatment with active CRT against inactive CRT; this overall estimate included patients with natural rhythm and patients with a prior definitive pacemaker, and even studies with implant devices with CRT + ICD functionalities in which, during the active period, the CRT function was deactivated. Subsequently the efficacy of the CRT was measured against OMT, both in prior pacemaker users and in patients without this device. Also the efficacy of CRT + ICD devices against ICD functionality only was evaluated. Finally, the efficacy of ICD devices against OMT was also measured.

A network of evidence or graphic representation was constructed using the efficacy of the compared treatments (OMT, CRT, ICD, and CRT+ICD). Direct estimates of the comparisons of the effect of various options versus OMT were obtained. On meeting the statistical consistency assumptions, the results of direct and indirect comparisons were added. We made a network meta-analysis with effect measures of direct comparisons in additive scale (log OR) and with its variances and covariances from a three-arm study $\left(\mathrm{COMPANION}^{12}\right)$, obtaining the net effect estimates using the Bucher method; we also performed a multivariate meta-regression to rank the benefit of these interventions in order.

\section{RESULTS}

With our search strategy we obtained 43 clinical trials involving 13017 patients, after ruling out those not addressing the aim of our study (among these were several comparative studies of different types of CRT), clinical trials in progress, observational studies, and meta-analyses (figure $1^{13}$ ).

The methodological quality of the studies obtained was low. The assessment of this aspect with the RevMan programme tool showed us that the majority of studies used an unsuitable or uncertain blinding of patients and clinicians when assessing the outcome, with unclear randomisation mechanisms and questionable concealment of the randomisation sequence (figure 2). Data from individual studies are shown in online supplementary figure A.

The features and the methodological quality of these studies are shown in the online supplementary appendix.

The overall estimate of effect on the active CRT strategy compared to the inactive CRT strategy, which includes the comparisons of CRT versus OMT and CRT + ICD versus ICD, is shown in figure 3 . There is an effect on the reduction of mortality which is statistically significant (OR 0.72 , 95\% CI 0.64 to 0.81). This outcome translates into a number needed to treat
Figure 2 Summary of the study quality assessment included in the methodology quality assessment kit of the Cochrane Collaboration (RevMan).
Random sequence generation (selection bias)

Allocation concealment (selection bias)

Blinding of participants and personnel (performance bias) Blinding of outcome assessment (detection bias) Incomplete outcome data (attrition bias) Low risk of bias Unclear risk of bias

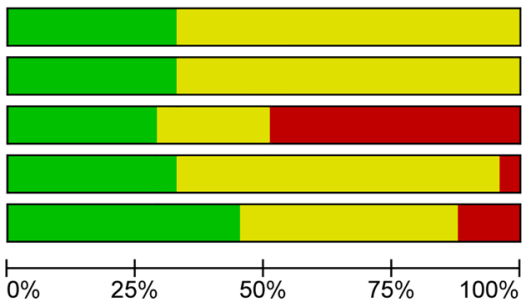

$\square$ High risk of bias 


\begin{tabular}{|c|c|c|c|c|c|c|c|c|}
\hline \multirow[b]{2}{*}{ Study or Subgroup } & \multicolumn{2}{|c|}{ Experimental } & \multicolumn{2}{|c|}{ Control } & \multirow[b]{2}{*}{ Weight } & \multirow{2}{*}{$\begin{array}{l}\text { Odds Ratio } \\
\text { M-H, Random, } 95 \% \mathrm{Cl}\end{array}$} & \multirow{2}{*}{\multicolumn{2}{|c|}{$\begin{array}{c}\text { Odds Ratio } \\
\text { M-H, Random, } 95 \% \text { CI }\end{array}$}} \\
\hline & Events & Total & Events & Total & & & & \\
\hline Abraham 2002 MIRACLE & 12 & 228 & 16 & 225 & $2.3 \%$ & $0.73[0.34,1.57]$ & $\rightarrow$ & \\
\hline Abraham2004 MIRACLEICDII & 2 & 85 & 2 & 101 & $0.3 \%$ & $1.19[0.16,8.65]$ & & \\
\hline Albertsen 2008 & 1 & 25 & 1 & 25 & $0.2 \%$ & $1.00[0.06,16.93]$ & & \\
\hline Beshai 2007 RethinQ & 5 & 87 & 2 & 85 & $0.5 \%$ & $2.53[0.48,13.42]$ & - & \\
\hline Brignole 2005 OPSITE & 2 & 28 & 4 & 28 & $0.4 \%$ & $0.46[0.08,2.75]$ & & \\
\hline Brignole 2011 APAF & 3 & 97 & 4 & 89 & $0.6 \%$ & $0.68[0.15,3.12]$ & & \\
\hline Bristow 2004 COMPANION & 131 & 617 & 77 & 308 & $13.2 \%$ & $0.81[0.59,1.12]$ & - & r \\
\hline Cazeau 2001 MUSTIC-SR & 1 & 58 & 2 & 58 & $0.2 \%$ & $0.49[0.04,5.57]$ & & \\
\hline Cleland 2005 CARE-HF & 101 & 409 & 154 & 404 & $15.1 \%$ & $0.53[0.39,0.72]$ & $=$ & \\
\hline Curtis 2013 BLOCK HF & 75 & 349 & 90 & 342 & $11.1 \%$ & $0.77[0.54,1.09]$ & - & \\
\hline Diab 2011 & 1 & 24 & 3 & 22 & $0.2 \%$ & $0.28[0.03,2.87]$ & & \\
\hline Doshi 2005 PAVE-VecToR & 13 & 146 & 19 & 106 & $2.4 \%$ & $0.45[0.21,0.95]$ & & \\
\hline Foley 2011 RESPOND & 6 & 31 & 10 & 29 & $1.0 \%$ & $0.46[0.14,1.48]$ & & \\
\hline Higgins 2003 CONTAK-CD & 11 & 245 & 16 & 245 & $2.2 \%$ & $0.67[0.31,1.48]$ & & - \\
\hline Kindermann 2006 HOBIPACE & 1 & 32 & 1 & 32 & $0.2 \%$ & $1.00[0.06,16.71]$ & & \\
\hline Leclerq 2002 MUSTIC-AF & 1 & 59 & 0 & 59 & $0.1 \%$ & $3.05[0.12,76.44]$ & & \\
\hline Leclerq 2007 RD-CHF & 2 & 56 & 4 & 56 & $0.5 \%$ & $0.48[0.08,2.74]$ & & \\
\hline Linde 2008 REVERSE & 9 & 419 & 3 & 191 & $0.8 \%$ & $1.38[0.37,5.14]$ & & \\
\hline Lozano2000 VENTAK/CONTAK & 5 & 222 & 10 & 222 & $1.2 \%$ & $0.49[0.16,1.45]$ & & \\
\hline Martinelli 2010 COMBAT & 2 & 60 & 13 & 60 & $0.6 \%$ & $0.12[0.03,0.58]$ & & \\
\hline Moss 2009 MADIT-CRT & 74 & 1089 & 53 & 731 & $10.2 \%$ & $0.93[0.65,1.34]$ & & \\
\hline Orlov 2010 AVAIL CLS/CRT & 8 & 129 & 2 & 34 & $0.5 \%$ & $1.06[0.21,5.23]$ & & \\
\hline Piepoli 2008 & 7 & 44 & 8 & 45 & $1.1 \%$ & $0.88[0.29,2.66]$ & & \\
\hline Pinter 2009 & 1 & 36 & 1 & 36 & $0.2 \%$ & $1.00[0.06,16.63]$ & & \\
\hline Pokushalov 2010 & 9 & 84 & 21 & 80 & $1.9 \%$ & $0.34[0.14,0.79]$ & & \\
\hline Res 2007 BRIGHT & 1 & 42 & 1 & 42 & $0.2 \%$ & $1.00[0.06,16.53]$ & & \\
\hline RHYTHM ICD 2004 & 9 & 119 & 3 & 59 & $0.8 \%$ & $1.53[0.40,5.87]$ & & \\
\hline Stockburger2011 PREVENTHF & 0 & 50 & 1 & 58 & $0.1 \%$ & $0.38[0.02,9.53]$ & & \\
\hline Tang 2010 RAFT & 186 & 894 & 236 & 904 & $28.5 \%$ & $0.74[0.60,0.93]$ & $=$ & \\
\hline Thibault 2013LESSER-EARTH & 2 & 44 & 1 & 41 & $0.2 \%$ & $1.90[0.17,21.84]$ & & \\
\hline van Geldorp 2010 & 1 & 37 & 0 & 37 & $0.1 \%$ & $3.08[0.12,78.14]$ & & \\
\hline Young 2003 MIRACLE ICD & 14 & 187 & 15 & 182 & $2.4 \%$ & $0.90[0.42,1.92]$ & & - \\
\hline Yu 2009 PACE & 3 & 89 & 4 & 88 & $0.6 \%$ & $0.73[0.16,3.37]$ & & \\
\hline Total $(95 \% \mathrm{Cl})$ & & 6121 & & 5024 & $100.0 \%$ & $0.72[0.64,0.81]$ & 1 & \\
\hline Total events & 699 & & 777 & & & & & \\
\hline $\begin{array}{l}\text { Heterogeneity: } \mathrm{Tau}^{2}=0.00 ; \mathrm{Chi}^{2} \\
\text { Test for overall effect: } Z=5.59(P\end{array}$ & $\begin{array}{l}26.09, \mathrm{df} \\
<0.00001\end{array}$ & ? $(\mathrm{P}$ & 76); $1^{2}$ & & & & $02 \quad 0.1$ & $1 \quad 10$ \\
\hline
\end{tabular}

Figure 3 Forest plot for assessing mortality with devices with cardiac resynchronisation therapy (CRT) 'on' versus CRT 'off'. OMT, optimal medical and pharmacological treatment.

(NNT) for mortality of 26 (95\% CI 20 to 37) for an average follow-up period of 21.1 months. No publication bias was detected: neither graphically (funnel plot-figure 4, Egger method-online supplementary figure B) nor using numerical methods (Rosenthal index, Gleser Olkin method). There is no statistical heterogeneity in the estimate of that effect (nonsignificant $\mathrm{Q}$ heterogeneity and $\mathrm{I}^{2}$, Galbraith plot). L'Abbé plot (figure 5) shows some degree of heterogeneity between studies with low mortality $(\mathrm{OR}=1)$ and studies with greater mortality (OR $<1$, protective effect).

Despite the shorter follow-up period, crossover studies show very similar results (OR, NNT) compared with non-crossover studies (see online supplementary figure $\mathrm{C}$ ).

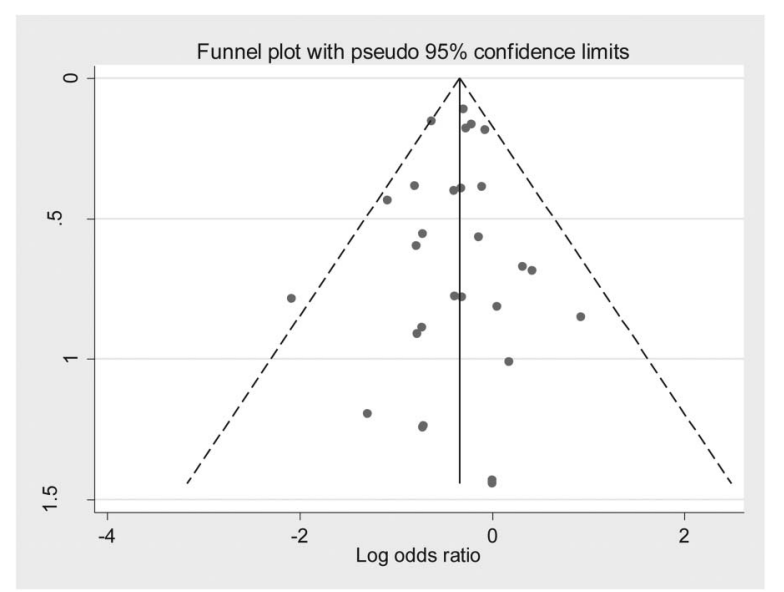

Figure 4 Funnel plot for assessing mortality in comparative studies of cardiac resynchronisation therapy (CRT) 'on' versus CRT 'off'.
Comparison of CRT versus OMT shows an OR for mortality of 0.65 (95\% CI 0.55 to 0.76 ), for a mean follow-up over 19 months, with an estimated NNT of 16 (95\% CI 11-23) with no apparent heterogeneity. Efficacy estimates for patients without previous pacemaker are somewhat more positive than the ones achieved in patients with previous implantation of a pacemaker (see online supplementary figure D).

Comparison of CRT + ICD versus ICD showed an OR for mortality of 0.81 (95\% CI 0.68 to 0.96 ) for a mean follow-up period of 22.82 months, with an NNT of 48 (95\% CI 29-214) (see online supplementary figure F). It was observed that the greater part of the benefit achieved depended on the RAFT ${ }^{14}$ study; being a long-term study, and with a population size

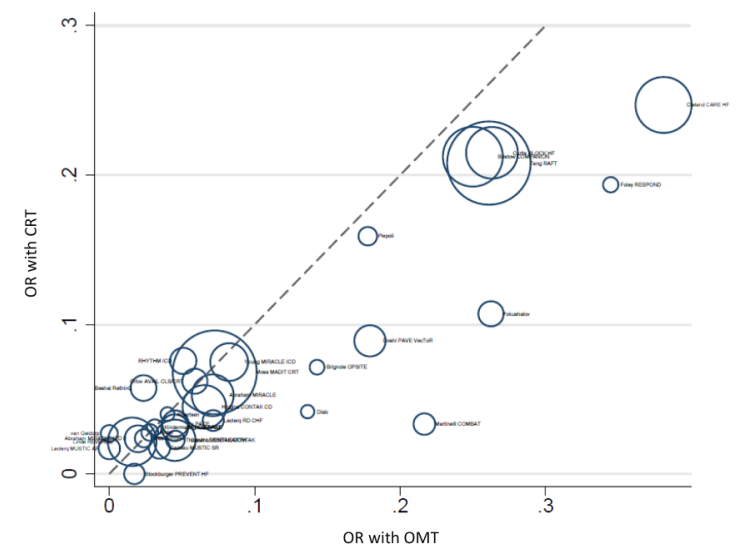

Figure 5 L'Abbé plot to assess the heterogeneity between studies with low and greater mortality. 


\section{Original research}

\section{electrical devices vs OMT}

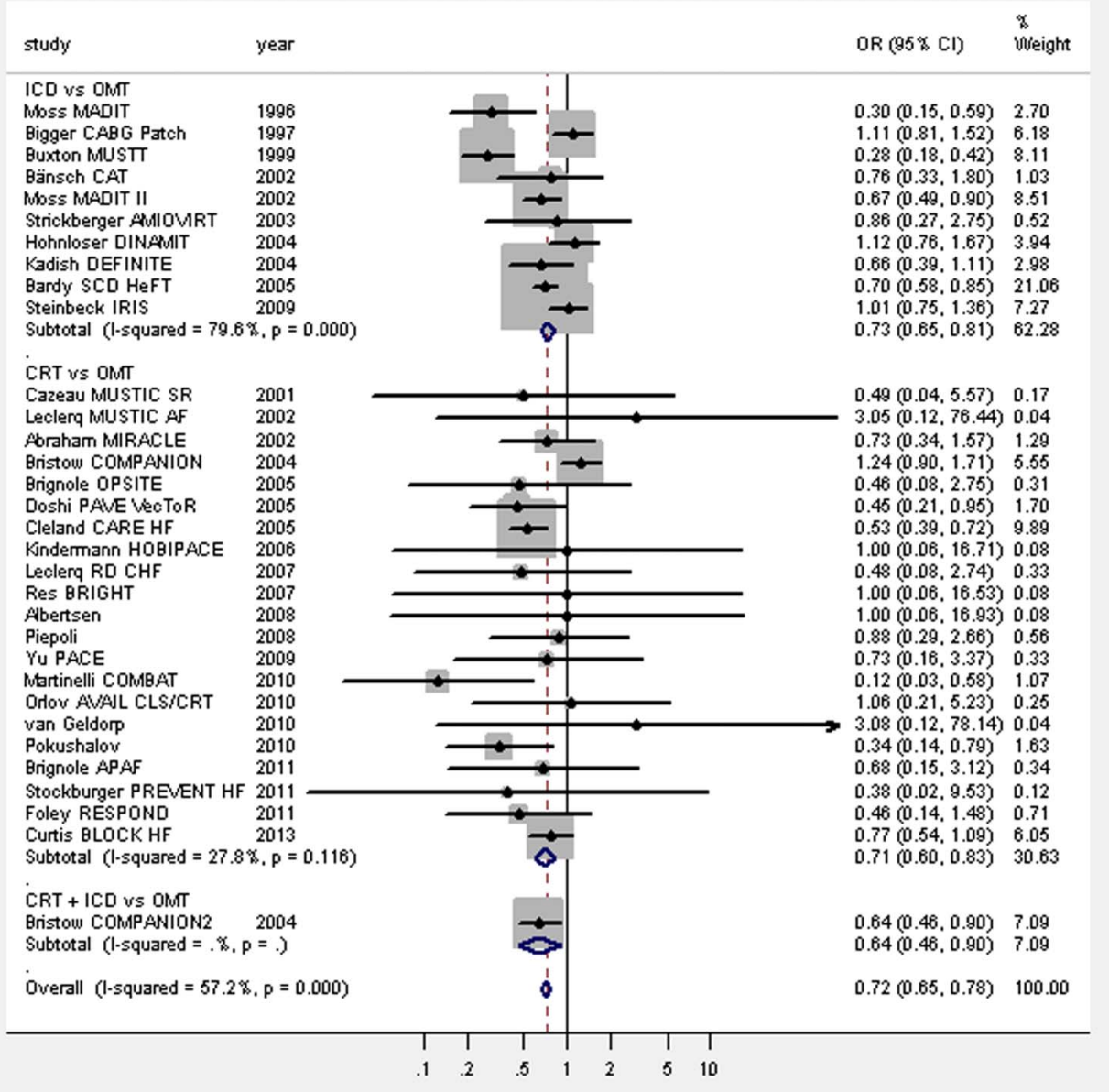

Figure 6 Comparison of the different electrical devices versus optimal medical and pharmacological treatment (OMT). CRT, cardiac resynchronisation therapy; ICD, implantable cardioverter defibrillator.

Figure 7 Results of comparisons of treatments in the network. The comparisons with continuous lines are direct. The indirect comparisons are shown with broken lines.

A comparison is mixed if it has continuous and broken lines. CRT, cardiac resynchronisation therapy; ICD, implantable cardioverter defibrillator; OMT, optimal medical and pharmacological treatment.

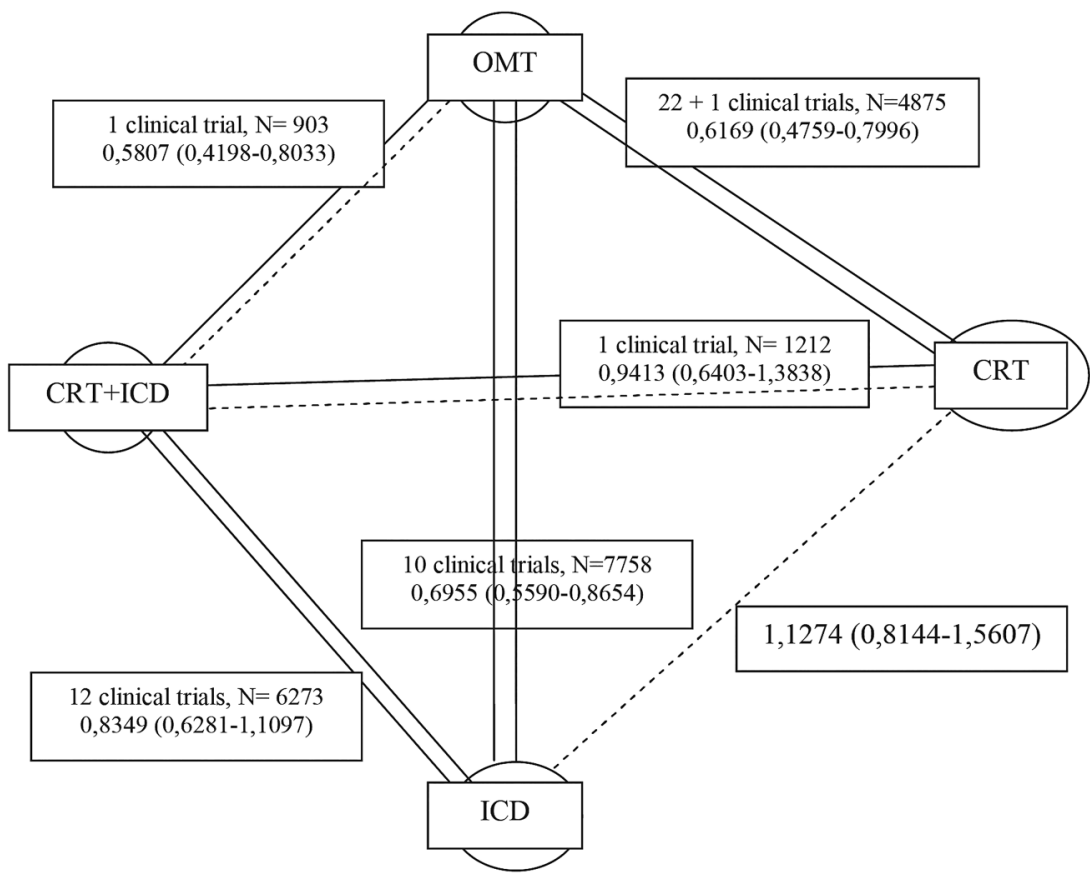




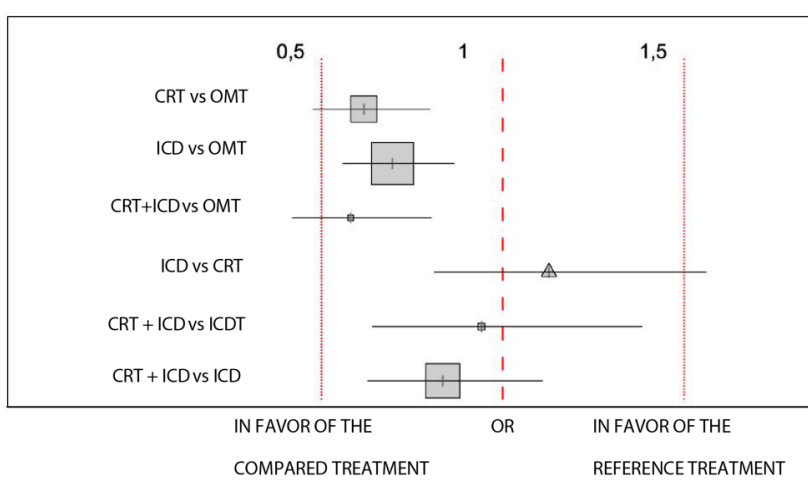

Figure 8 Graphical representation of the relative efficacy of the different compared therapeutic options. CRT, cardiac resynchronisation therapy; ICD, implantable cardioverter defibrillator; OMT, optimal medical and pharmacological treatment.

considerably higher than the rest of this comparison, the estimate of the proposed effect was maintained.

Comparison of ICD versus OMT showed an OR for mortality of 0.69 (95\% CI 0.52 to 0.92 ) for a mean follow-up period of 46.58 months, with an estimated NNT of 18 (95\% CI 13-26) (see online supplementary figure G). Statistical heterogeneity (with $\mathrm{Q}$ and $\mathrm{I}^{2}$ significant) and clinical heterogeneity (inclusion of varied profile of patients) were observed; therefore the estimate in a specific group of patients could be more benevolent than that which the group achieved. Methodological heterogeneity of the MUSTT ${ }^{15}$ study was assessed. It was finally decided to maintain that initial efficacy estimate.

A comparison of all treatments with electrical devices versus OMT can be seen in figure 6 (and online supplementary figure E). There is statistical heterogeneity in the different strategies compared to each other, and within each comparison (CRT vs OMT, ICD vs OMT, and CRT + ICD vs OMT).

Other direct comparisons obtained were based on the COMPANION study outcomes. ${ }^{12}$ This study was presented as a three-arm study (CRT, CRT+ICD, and OMT) with two comparisons: CRT versus OMT, and CRT+ICD versus OMT. The estimate of the effect of CRT +ICD versus OMT showed an OR for mortality of 0.64 (95\% CI 0.46 to 0.90 ) after 12 months. The comparison of CRT+ICD versus CRT is not provided for in the statistical approach of the study, but we can assess it as an approximation for our network meta-analysis (OR 0.79, 95\% CI 0.60 to 1.06 ; also after 12 months).

Subsequently, a network meta-analysis was performed, combining the direct comparisons, obtained in clinical trials, with indirect comparisons between studies with a common comparator, in order to attempt to establish a treatment efficacy order. Multivariate meta-regression was undertaken, calculating the effect measurements, their variances, and the covariance of the three-arm study. A matrix of variances and covariances was created, and parameters of indirect and mixed comparisons were calculated. There is no inconsistency in the design of each comparison of treatments, and there is also no global (across the network) inconsistency.

Figures 7 and 8 and table 1 show the effect of the different therapeutic comparisons one by one. The online supplementary figure $\mathrm{H}$ shows the different studies included in each branch.

We established the effectiveness of the devices with multivariate meta-regression. OMT, the option with greater OR mortality rate, was the least effective, and the other options with minor OR had greater effectiveness. The least effective device was ICD (70.1\% probability of achieving greater OR), followed by CRT $(22.6 \%)$, and finally the best option was the combination therapy CRT+ICD (7.3\% probability). However, the OR estimates at 95\% CIs overlap considerably, so that categorical conclusions cannot be reached on the comparative effectiveness of these treatments, and the order of efficacy is just a guideline for future trials.

\section{DISCUSSION}

The results of our study are clear. All the electrical devices assessed are better than the best medical treatment, since they achieve a reduction in mortality. In the comparisons with OMT, and the comparisons between them, there is considerable overlapping in the OR estimates for mortality by CI, although the outcomes would appear to be better with CRT+ICD in first place, CRT in second place, and ICD in last place. This visual order was verified by our simulation. Such superimposed estimates lead us to the conclusion that it cannot be categorically stated that one option provides a clear reduction in mortality over another.

The results of our network meta-analysis is similar to those in the study by Lam and Owen, ${ }^{9}$ developed under Bayesian methodology. Despite there being no intention to compare works with different methodologies, and despite the inclusion of different studies, the results are similar-that is, the superiority of the combined therapy CRT + ICD was observed, with a significant degree of overlapping of the CIs. Neither our study nor that of Lam and Owen ${ }^{9}$ can support the indiscriminate use of CRT +ICD combined therapy in our spectrum of patients.

We assessed for publication bias by trial funding, but both graphical (funnel plot, Egger) and numerical methods (Begg, Rosenthal) showed that publication bias was unlikely. Although not the primary outcome, mortality was reported in all studies included in our meta-analysis. We specifically followed unpublished completed trials mentioned in ClinicalTrials.gov.

We can reasonably rule out the effect of small studies. It could be argued that our mathematical approach (random effects model) gives too much value to the results of small studies. Repeating the calculations comparing the effects of active CRT strategy versus inactive CRT with a fixed effects

Table 1 Comparison of the different options; the compared option is in the rows and the reference option in the columns

\begin{tabular}{|c|c|c|c|c|}
\hline & ОМт & CRT & ICD & $C R T+I C D$ \\
\hline ОМт & $x x X$ & $X X X$ & $x X X$ & $X X X$ \\
\hline CRT & $0.616(0.475$ to 0.799$)$ & $x X X$ & $x x x$ & $x X X$ \\
\hline ICD & 0.695 (0.559 to 0.865$)$ & 1.127 (0.814 to 1.56$)$ & $x x x$ & $x x x$ \\
\hline$C R T+I C D$ & $0.580(0.419$ to 0.803$)$ & 0.94 (0.64 to 1.383$)$ & 0.834 (0.628 to 1.109$)$ & $x x x$ \\
\hline
\end{tabular}


model gives estimates that are virtually identical (OR $0.71,95 \%$ CI 0.63 to 0.80 ) to the estimates achieved with the random effects model.

In view of the published clinical trials, meta-analyses and observational studies, and the unlikely publication bias, it can be concluded that electrical therapies are effective in patients with HF. The update of the review (2013) does not reduce the validity of its conclusions, showing that their findings are consistent and clear, and unlikely to change with new studies. Since the benefits were greater than the risks, the available evidence was of high quality, and the costs acceptable, we are in a position to recommend the use of these devices. ${ }^{13} 16$

The general purpose of a network meta-analysis was to compare different interventions that would be considered plausible solutions to a problem. The devices can be implanted for different reasons in probably different populations (definitive pacemaker with added resynchronisation function, ICD for primary prevention of SCD). We have developed this work as a comparison of different therapeutic strategies to a patient at risk of death from HF and arrhythmia. The mechanisms of the compared devices are different, but the profile of the patient is common. Depressed LVEF is a risk factor of SCD, and as these two problems coexist in these patients, it may make sense to compare CRT and ICD even though their mechanisms are different. Several subgroup analyses were made, and all of these showed efficacy of CRT, so it may be plausible to make the overall estimate of effect, remembering that in the classic indication (sinus rhythm, depressed LVEF, wide QRS) the benefit is greater.

There are authors who believe that indirect comparisons may over- or underestimate the effects of treatments compared with the limitations obtained from direct comparisons. Indirect comparisons based on potentially imperfect direct comparisons may contribute more biased data than those obtained from classic meta-analysis based on direct comparisons, due to lack of

\section{Key messages}

What is already known about this subject?

- CRT devices are effective in patients with moderate to severe heart failure and depressed left ventricular ejection fraction.

- ICD devices are effective in primary prevention of SCD in patients with symptomatic heart failure.

- There are no studies comparing directly CRT + ICD versus CRT.

\section{What does this study add?}

- Unlike previous studies, this meta-analysis, which compares the use of different electrical devices in the treatment of patients with heart failure and risk of sudden death, includes a large number of studies and deterministic methodology.

- CRT benefit is greater in patients with higher mortality.

- Despite the apparent benefit found with the combined TRC +DAl option, no recommendation is possible due to the strong overlapping of confidence intervals.

\section{How might this impact on clinical practice?}

- The need to carry out further studies should be assessed, comparing directly CRT+ICD versus CRT.

- The placement of CRT devices in patients with increased risk of mortality should be recommended. homogeneity, publication bias, selection bias, etc. However, network meta-analysis may be a useful exploratory tool in yet undeveloped research fields, faced with alternatives of unconsidered versus provided treatments in practice, or support for economic assessment studies of these treatments. The absence of studies that directly compare CRT +ICD with CRT may be due to the lack of interest from the pharmaceutical industry in undertaking such studies, but also to the ethical dilemma involved in not adding ICD treatment to patients in whom there is a potentially arrhythmogenic substrate.

Therefore, and in the absence of direct comparisons of several therapeutic strategies in this field, network meta-analysis arises as a new paradigm for evidence, accepted by governmental agencies such as the National Institute for Health and Care Excellence (NICE) ${ }^{7}$

Acknowledgements Our thanks to Joaquin Primo, gastroenterologist of the Sagunto hospital; Manuel Arranz, from the library of the Valencian School of Health Studies (EVES); Rosario García, from the library of the Faculty of Medicine of the University of Valencia; Ferran Catalá, from the Division of Pharmacoepidemiology and Pharmacovigilance of the Spanish Medicament Agency (AEMPS); and Marta Quilis, internist of the Sagunto Hospital for their help in carrying out this work. Also our thanks to Niall O'cinsealach and María Quejada for their collaboration in correction of grammatical errors and translating this text. And special thanks to Alberto García for his help with the editing of the images.

Contributors All authors contribute to the development of the work. MAGG and MARA conducted the peer-review process, AMC and MAGG translated the text into English, and all five authors reviewed the methodology aspects, the results and the conclusions of the work.

Competing interests RRG is section chief of the cardiac electrophysiology section in Clinic Hospital; he has participated in clinical trials of CRT and ICD, and has lectured conferences funded by Medtronic and St Jude companies. The other authors declare no conflict of interests.

Provenance and peer review Not commissioned; externally peer reviewed.

\section{REFERENCES}

1 García García MA, Rosero Arenas MA, Ruiz Granell R, et al. Eficacia de la resincronización cardiaca en el tratamiento de la insuficiencia cardiaca sintomática. Revisión sistemática y metaanálisis. Av Cardiol 2015;35:67-75.

2 Díaz Infarte E, Hernández Madrid A, Brugada-Terradellas J, et al. Consenso sobre indicaciones de resincronización cardiaca del grupo de Resincronización Cardiaca de la Sociedad Española de Cardiología. Rev Esp Cardiol 2005;5(Suppl I):03B-11B.

3 Vardas PE, Auricchio A, Blanc JJ, et al. Guidelines for cardiac pacing and cardiac resynchronization therapy: the Task Force for Cardiac Pacing and Cardiac Resynchronization Therapy of the European Society of Cardiology. Developed in collaboration with the European Heart Rhythm Association. Eur Heart J 2007:28:2256-95.

4 Dorian P. Effective and efficient use of implantable defibrillators: sometimes it's over when it's over. CMAJ 2009;180:599-600

5 Kannel WB, Wilson PW, D'Agostino RB, et al. Sudden coronary death in women. Am Heart J 1998; 136:205-12.

6 Mosterd A, Cost B, Hoes AW, et al. The prognosis of heart failure in the general population: the Rotterdam Study. Eur Heart J 2001;22:1318-27.

7 Catalá-López F, Tobías A. Evidencia clínica procedente de comparaciones indirectas y mixtas: algunas consideraciones prácticas. Farm Hosp 2012;36:125-32.

8 Catalá-López F, Tobías A. Síntesis de la evidencia clínica y metaanálisis en red con comparaciones indirectas. Med Clin 2013;140:182-7.

9 Lam SK, Owen A. Combined resynchronisation and implantable defibrillator therapy in left ventricular dysfunction: Bayesian network meta-analysis of randomised controlled trials. BMJ 2007;335:925

10 Urrútia G, Bonfill X. Declaración PRISMA: una propuesta para mejorar la publicación de revisiones sistemáticas y metaanálisis. Med Clin 2010;135:507-11.

11 Jadad AR, Moore RA, Carroll D, et al. Assessing the quality of reports of randomized clinical trials: is blinding necessary? Control Clin Trials 1996;17:1-12.

12 Bristow MR, Saxon LA, Boehmer J, et al, Comparison of Medical therapy, Pacing and Defibrillation in Heart Failure (COMPANION) Investigators. Cardiac-resynchronization therapy with or without an implantable defibrillator in advanced chronic heart failure. N Engl J Med 2004;350:2140-50.

13 Doctoral thesis presented at the Valencia University in 2014. "Eficacia de la resincronización cardiac en el tratamiento de la insuficiencia cardiaca sintomática. Metaanálisis y análisis económico". http://www.google.es/url?url=http://roderic.uv. 


\section{Original research}

es/bitstream/handle/10550/37137/TESIS\%2520DOCTORALmiguelangelgarcia.pdf\% 3Fsequence\%3D1\&rct=j\&frm=1\&q=\&esrc=s\&sa=U\&ei=6UrzU9_kCuyW0QXYuo DgCg\&ved=0CBQQFjAA\&sig2=QqmeempfFijb_jbvegCQBg\&usg=AFQjCNEy8vj4Yr NBH-jA3JaQOf7peSudEA)

14 Tang AS, Wells GA, Talajic M, et al. Resynchronization-Defibrillation for Ambulatory Heart Failure Trial (RAFT) Investigators. Cardiac-resynchronization therapy for mild-to-moderate heart failure. N Engl J Med 2010;363:2385-95.
15 Buxton $A E$, Lee $K L$, Fisher JD, et al. A randomized study of the prevention of sudden death in patients with coronary artery disease. Multicenter Unsustained Tachycardia Trial (MUSTT) Investigators. N Engl J Med 1999;341:1882-90. Erratum in: N Engl J Med 2000;342:1300.

16 Marzo-Castillejo M, Alonso-Coello P. Clasificación de la calidad de la evidencia y fuerza de las recomendaciones. GRADE Working Group. Aten Primaria 2006;37:40-50. 